\title{
Model-based Prognostics under Limited Sensing
}

\author{
Matthew Daigle* and Kai Goebel ${ }^{\dagger}$ \\ *University of California, Santa Cruz, NASA Ames Research Center, Moffett Field, CA, USA \\ $\dagger$ NASA Ames Research Center, Moffett Field, CA, USA \\ $\{$ matthew.j.daigle,kai.goebel\}@nasa.gov
}

\begin{abstract}
Prognostics is crucial to providing reliable conditionbased maintenance decisions. To obtain accurate predictions of component life, a variety of sensors are often needed. However, it is typically difficult to add enough sensors for reliable prognosis, due to system constraints such as cost and weight. Model-based prognostics helps to offset this problem by exploiting domain knowledge about the system, its components, and how they fail by casting the underlying physical phenomena in a physicsbased model that is derived from first principles. We develop a model-based prognostics methodology using particle filters, and investigate the benefits of a model-based approach when sensor sets are diminished. We apply our approach to a detailed physicsbased model of a pneumatic valve, and perform comprehensive simulation experiments to demonstrate the robustness of modelbased approaches under limited sensing scenarios using prognostics performance metrics.
\end{abstract}

\section{INTRODUCTION}

Prognostics is a key enabling technology for applying condition-based maintenance. The goal of prognostics is to make end of life (EOL) and remaining useful life (RUL) predictions that enable timely maintenance decisions to be made. As with diagnostics, prognostics methods may typically be categorized as either data-driven or model-based. Datadriven approaches do not take advantage of system and domain knowledge, but instead use learning methods to identify trends and determine EOL and RUL [1]. Such methods rely on large amounts of run-to-failure data that are used to train the algorithms. The training becomes dependent on the set of sensors used, and, therefore, is not robust to loss of sensors or a possible lack of sensors. Furthermore, the required training data, in many cases, does not exist. In contrast, modelbased approaches exploit domain knowledge of the system, its components, and how they fail in order to provide EOL and RUL predictions [2]-[5]. The underlying physical phenomena are captured in a physics-based model that is derived from first principles, therefore, model-based approaches can provide EOL and RUL estimates that are much more accurate and precise than data-driven approaches, if the models are correct. In addition, model-based approaches can be robust to sensor loss and still work under limited sensing environments with an accurate model.

In this paper, we demonstrate the effectiveness of modelbased approaches under limited sensing conditions. Reduction of the number of sensors implies an increase in uncertainty. Other sources of uncertainty are also present, such as model inaccuracies, sensor noise, and uncertainty in future input profiles. To help manage this uncertainty, we apply a particle filtering approach that is based on joint state-parameter estimation. Particle filters approximate the posterior probability distribution as a set of discrete, weighted samples. Although suboptimal, the advantage of particle filters is that they can be applied to systems which may be nonlinear and have nonGaussian noise terms, where optimal solutions are unavailable or intractable. Particle filtering approaches have previously been successful in prognostics applications. In [4], the authors apply a particle filtering approach to prediction of end of discharge and EOL in lithium-ion batteries, which highlights the performance that can be achieved by having accurate models. In [6], the authors apply a particle filter-based prognosis method to prediction of battery grid corrosion. Both [7] and [8] apply particle filtering to estimation and prediction of crack growth. Performance improvements over standard particle filter methods can be achieved in prognostics with advanced filtering techniques, such as the use of correction loops [9], fixed-lag filters [5], Rao-Blackwellized particle filters [10], [11], and risk-sensitive particle filters [12].

In this paper, we develop a model-based prognostics framework that incorporates particle filters. As a case study, we construct a detailed physics-based model of a pneumatic valve that includes models of different damage mechanisms. We run a comprehensive set of prognostics experiments in simulation to demonstrate the robustness of the approach to limited sensor sets, evaluated using established prognostic metrics [13], [14].

The paper is organized as follows. Section II formulates the prognostics problem and overviews the computational architecture we adopt. Section III develops the damage estimation method using particle filters. Section IV describes the prediction procedure. Section $\mathrm{V}$ presents the pneumatic valve case study with experimental results in simulation. Section VI concludes the paper.

\section{Prognostics ApProACH}

\section{A. Problem Formulation}

The problem of prognostics is to predict the EOL and/or the RUL of a component. In this paper, we develop a general model-based approach, where the system model is given by

$$
\begin{aligned}
& \dot{\mathbf{x}}(t)=\mathbf{f}(t, \mathbf{x}(t), \boldsymbol{\theta}(t), \mathbf{u}(t), \mathbf{v}(t)) \\
& \mathbf{y}(t)=\mathbf{h}(t, \mathbf{x}(t), \boldsymbol{\theta}(t), \mathbf{u}(t), \mathbf{n}(t))
\end{aligned}
$$

where $\mathbf{x}(t) \in \mathbb{R}^{n_{x}}$ is the state vector, $\boldsymbol{\theta}(t) \in \mathbb{R}^{n_{\theta}}$ is the parameter vector, $\mathbf{u}(t) \in \mathbb{R}^{n_{u}}$ is the input vector, $\mathbf{v}(t) \in \mathbb{R}^{n_{v}}$ is the process noise vector, $\mathbf{f}$ is the state equation, $\mathbf{y}(t) \in \mathbb{R}^{n_{y}}$ 


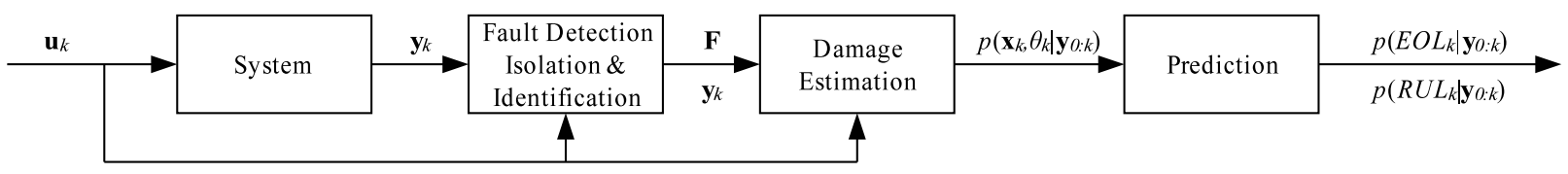

Fig. 1. Prognostics architecture.

is the output vector, $\mathbf{n}(t) \in \mathbb{R}^{n_{n}}$ is the measurement noise vector, and $\mathbf{h}$ is the output equation.

Our goal is to predict EOL at a given time point $t_{P}$ using the discrete sequence of observations up to time $t_{P}$, denoted as $\mathbf{y}_{0: t_{P}}$. In order to determine when EOL has been reached, we require a condition that is a function of the system state and parameters, $C_{E O L}(\mathbf{x}(t), \boldsymbol{\theta}(t))$, which determines whether EOL has been reached, where

$$
C_{E O L}(\mathbf{x}(t), \boldsymbol{\theta}(t))= \begin{cases}1, & \text { if EOL is reached } \\ 0, & \text { otherwise. }\end{cases}
$$

Using this function, we can define EOL with

$$
E O L\left(t_{P}\right) \triangleq \underset{t \geq t_{P}}{\arg \min } C_{E O L}(\mathbf{x}(t), \boldsymbol{\theta}(t))=1
$$

and RUL with

$$
R U L\left(t_{P}\right) \triangleq E O L\left(t_{P}\right)-t_{P}
$$

Because of the noise inherent in the process, the measurements, and future inputs, we must compute a probability distribution of the EOL or RUL, i.e., the goal is to compute, at time $t_{P}, p\left(E O L\left(t_{p}\right) \mid \mathbf{y}_{0: t_{P}}\right)$ or $p\left(R U L\left(t_{P}\right) \mid \mathbf{y}_{0: t_{P}}\right)$.

\section{B. Prognostics Architecture}

We adopt a model-based approach, wherein we develop detailed physics-based models of components and systems that include descriptions of how fault parameters evolve in time. These models depend on unknown and possibly timevarying wear parameters, $\boldsymbol{\theta}(t)$. Therefore, our solution to the prognostics problem takes the perspective of joint stateparameter estimation. In discrete time $k$, we estimate $\mathbf{x}_{k}$ and $\boldsymbol{\theta}_{k}$, and use these estimates to predict EOL and RUL at desired time points.

We employ the prognostics architecture in Fig. 1. The system is provided with inputs $\mathbf{u}_{k}$ and provides measured outputs $\mathbf{y}_{k}$. The fault detection, isolation, and identification (FDII) module provides a fault set $\mathbf{F}$, which is used by the damage estimation module to determine estimates of the states and unknown parameters, represented as a probability distribution $p\left(\mathbf{x}_{k}, \boldsymbol{\theta}_{k} \mid \mathbf{y}_{0: k}\right)$. This distribution is used by the prediction module which computes EOL and RUL using hypothesized future inputs. EOL and RUL are computed as probability distributions $p\left(E O L_{k_{P}} \mid \mathbf{y}_{0: k_{P}}\right)$ and $p\left(R U L_{k_{P}} \mid \mathbf{y}_{0: k_{P}}\right)$. In this paper, we focus on the damage estimation and prediction modules, and assume a solution to FDII.

\section{DAmAGE ESTIMATION}

To estimate the damage, we need to estimate $p\left(\mathbf{x}_{k}, \boldsymbol{\theta}_{k} \mid \mathbf{y}_{0: k}\right)$. In this paper, we use the particle filter for this purpose [15], [16]. With particle filters, the state distribution is approximated by a set of discrete weighted samples, or particles, $\left\{\left(\mathbf{x}_{k}^{i}, \boldsymbol{\theta}_{k}^{i}\right), w_{k}^{i}\right\}_{i=1}^{N}$, where $N$ denotes the number of particles, and for particle $i, \mathbf{x}_{k}^{i}$ denotes the state estimates, $\boldsymbol{\theta}_{k}^{i}$ denotes the parameter estimates, and $w_{k}^{i}$ denotes the weight.

Particle filters are best suited to estimation in nonlinear systems with possibly non-Gaussian noise, where optimal solutions are unavailable or intractable. In this respect, they may be viewed as a general (suboptimal) solution to the state estimation problem. Performance can be improved by increasing the number of particles, but this also results in higher computational costs. The number of particles must be chosen to suit the application requirements.

We employ the sampling importance resampling (SIR) particle filter, and implement the resampling step using systematic resampling [17]. In particle filters, the posterior density is approximated by

$$
p\left(\mathbf{x}_{k}, \boldsymbol{\theta}_{k} \mid \mathbf{y}_{0: k}\right) \approx \sum_{i=1}^{N} w_{k}^{i} \delta_{\left(\mathbf{x}_{k}^{i}, \boldsymbol{\theta}_{k}^{i}\right)}\left(d \mathbf{x}_{k} d \boldsymbol{\theta}_{k}\right),
$$

where $\delta_{\left(\mathbf{x}_{k}^{i}, \boldsymbol{\theta}_{k}^{i}\right)}\left(d \mathbf{x}_{k} d \boldsymbol{\theta}_{k}\right)$ denotes the Dirac delta function located at $\left(\mathbf{x}_{k}^{i}, \boldsymbol{\theta}_{k}^{i}\right)$.

Here, the parameters $\boldsymbol{\theta}_{k}$ evolve by some unknown random process that is independent of the state $\mathbf{x}_{k}$. To perform parameter estimation within a particle filter framework, however, we need to assign some type of evolution to the parameters. The typical solution is to use a random walk, i.e., for parameter $\theta, \theta_{k}=\theta_{k-1}+\xi_{k-1}$, where $\xi_{k-1}$ is typically Gaussian noise. With this type of evolution, during the sampling step particles are generated with parameter values that will be different from the initial guesses for the unknown parameters. The particles with parameter values closest to the true values should be assigned higher weight, thus allowing the particle filter to converge to the true values. The selected variance of the random walk noise must be large enough so as to allow convergence in a reasonable amount of time, but small enough such that when convergence is reached, the parameter can be tracked smoothly. Since the parameter values are unknown to start with, this can be a difficult task, but knowledge of the correct order of magnitude of the parameter is helpful. Additionally, correction loop methods can be used to tune this value online as a function of performance [9].

The pseudocode for a single step of the SIR filter is shown as Algorithm 1. Each particle is propagated forward to time $k$ by first sampling new parameter values and sampling new 


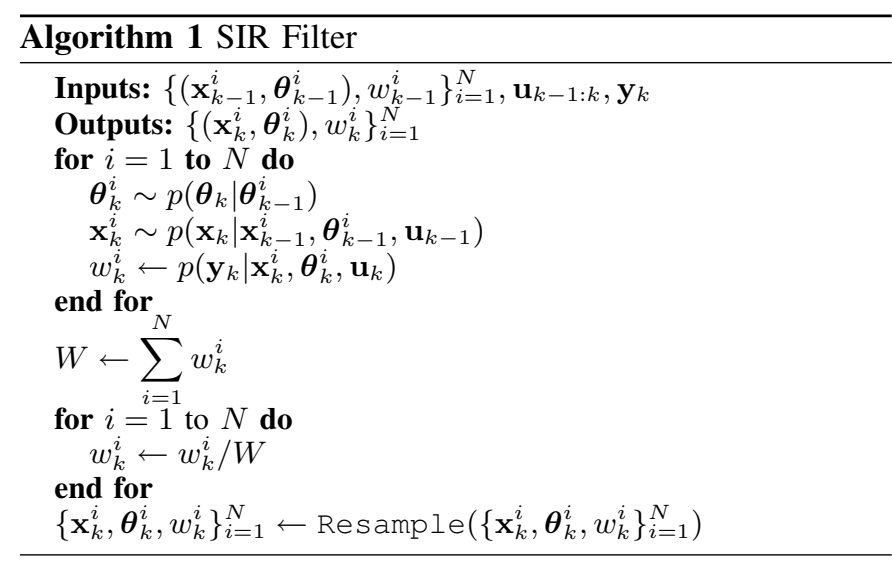

states. The particle weight is assigned using $\mathbf{y}_{k}$. The weights are then normalized, followed by the resampling step (see [15] for pseudocode of the systematic resampling algorithm).

\section{PREDiction}

In the prediction phase, we wish to compute at time $k_{P}$, $p\left(E O L_{k_{P}} \mid \mathbf{y}_{0: k_{P}}\right)$ and $p\left(R U L_{k_{P}} \mid \mathbf{y}_{0: k_{P}}\right)$. The particle filter computes

$$
p\left(\mathbf{x}_{k_{P}}, \boldsymbol{\theta}_{k_{P}} \mid \mathbf{y}_{0: k_{P}}\right) \approx \sum_{i=1}^{N} w_{k_{P}}^{i} \delta_{\left(\mathbf{x}_{k_{P}}^{i}, \boldsymbol{\theta}_{k_{P}}^{i}\right)}\left(d \mathbf{x}_{k_{P}} d \boldsymbol{\theta}_{k_{P}}\right) .
$$

We can approximate a prediction distribution $n$ steps forward as [18]

$$
\begin{aligned}
& p\left(\mathbf{x}_{k_{P}+n}, \boldsymbol{\theta}_{k_{P}+n} \mid \mathbf{y}_{0: k_{P}}\right) \approx \\
& \sum_{i=1}^{N} w_{k_{P}}^{i} \delta_{\left(\mathbf{x}_{k_{P}+n}^{i}, \boldsymbol{\theta}_{k_{P}+n}^{i}\right)}\left(d \mathbf{x}_{k_{P}+n} d \boldsymbol{\theta}_{k_{P}+n}\right) .
\end{aligned}
$$

So, for a particle $i$ propagated $n$ steps forward (without new data), we can simply take its weight as $w_{k_{P}}^{i}$. Similarly, we can approximate the EOL as

$$
p\left(E O L_{k_{P}} \mid \mathbf{y}_{0: k_{P}}\right) \approx \sum_{i=1}^{N} w_{k_{P}}^{i} \delta_{E O L_{k_{P}}^{i}}\left(d E O L_{k_{P}}\right) .
$$

To compute EOL, then, we propagate each particle forward to its own EOL and use that particle's weight at $k_{P}$ for the weight of its EOL prediction.

The pseudocode for the prediction procedure is given as Algorithm 2. Each particle $i$ is propagated forward until $C_{E O L}\left(\mathbf{x}_{k}^{i}, \boldsymbol{\theta}_{k}^{i}\right)$ evaluates to 1 , at this point EOL has been reached for this particle. Prediction requires hypothesizing future inputs of the system, $\hat{\mathbf{u}}_{k}$. The inputs must be chosen carefully because different inputs often have different effects on damage progression. If future inputs are unknown, inputs for each particle may be selected based on a distribution of possible anticipated input values.

\section{Case Study}

In order to illustrate our prognostics methodology, we take a pneumatic valve as a case study. We develop a physicsbased model of the valve and its damage mechanisms. We
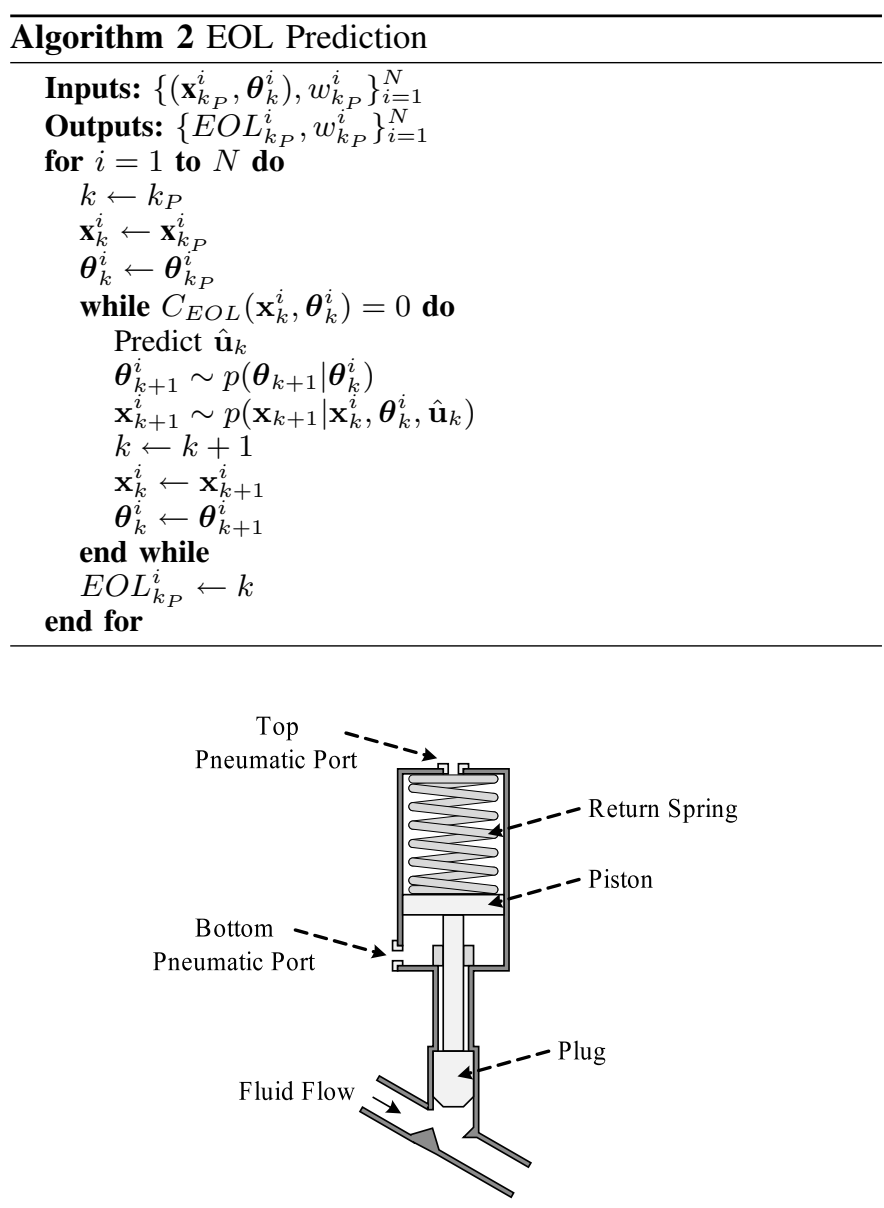

Fig. 2. Pneumatic valve.

then present simulation experiments to demonstrate joint stateparameter estimation and EOL/RUL prediction for different sets of sensors and noise levels. The scenarios are compared using prognostics performance metrics established in [13], [14].

\section{A. Component Modeling}

Pneumatic valves are complex mechanical systems used in many domains. These valves are actuated by gas, and can use different types of actuators. A normally-closed valve with a linear cylinder actuator is depicted in Fig. 2. The valve is opened by filling the chamber below the piston with gas up to the supply pressure, and evacuating the chamber above the piston down to atmospheric pressure. The valve is closed by filling the chamber above the piston, and evacuating the chamber below the piston. The return spring ensures that when pressure is lost, the valve will close due to the force exerted by the return spring.

We develop a physics model of the valve based on mass and energy balances. The system state includes the position of the valve, $x(t)$, the velocity of the valve, $v(t)$, the mass of the gas in the volume above the piston, $m_{t}(t)$, and the mass 
of the gas in the volume below the piston, $m_{b}(t)$ :

$$
\mathbf{x}(t)=\left[\begin{array}{c}
x(t) \\
v(t) \\
m_{t}(t) \\
m_{b}(t)
\end{array}\right] .
$$

The position when the valve is fully closed is defined as $x=0$. The stroke length of the valve is denoted by $L_{s}$; when the valve is fully open its position is $x=L_{s}$.

The derivatives of the states are described by

$$
\dot{\mathbf{x}}(t)=\left[\begin{array}{c}
v(t) \\
\frac{1}{m} \sum_{f} F(t) \\
f_{t}(t) \\
f_{b}(t)
\end{array}\right],
$$

where $m$ is the combined mass of the piston and plug, $\sum F(t)$ is the sum of forces acting on the valve, and $f_{t}(t)$ and $f_{b}(t)$ are the mass flows going into the top and bottom pneumatic ports, respectively.

The inputs are considered to be

$$
\mathbf{u}(t)=\left[\begin{array}{c}
p_{l}(t) \\
p_{r}(t) \\
u_{t}(t) \\
u_{b}(t)
\end{array}\right],
$$

where $p_{l}(t)$ and $p_{r}(t)$ are the fluid pressures on the left and right side of the plug, respectively, and $u_{t}(t)$ and $u_{b}(t)$ are the input pressures to the top and bottom pneumatic ports. These pressures will alternate between the supply pressure and atmospheric pressure depending on the commanded valve position.

The sum of forces acting on the piston includes (1) the forces from the pneumatic gas: $\left(p_{b}(t)-p_{t}(t)\right) A_{p}$, where $p_{b}(t)$ and $p_{t}(t)$ are the gas pressures on the bottom and the top of the piston, respectively, and $A_{p}$ is the surface area of the piston, (2) the forces from the fluid flowing through the valve: $\left(p_{r}(t)-p_{l}(t)\right) A_{v}$, where $A_{v}$ is the area of the valve contacting the fluid, (3) the weight of the moving parts of the valve: $-m g$, where $g$ is the acceleration due to gravity, (4) the spring force: $-k\left(x(t)-x_{o}\right)$, where $k$ is the spring constant and $x_{o}$ is the amount of spring compression when the valve is closed, (5) friction: $-r v(t)$, where $r$ is the coefficient of kinetic friction, and (6) the contact forces at the boundaries of the valve motion:

$$
\begin{cases}k_{c}(-x), & x<0 \\ 0, & 0 \leq x \leq L_{s} \\ -k_{c}\left(x-L_{s}\right), & x>L_{s},\end{cases}
$$

where $k_{c}$ is the (large) spring constant associated with the flexible seals.

The pressures $p_{t}(t)$ and $p_{b}(t)$ are calculated as:

$$
\begin{aligned}
p_{t}(t) & =\frac{m_{t}(t) R_{g} T}{V_{t_{0}}+A_{p}\left(L_{s}-x(t)\right)} \\
p_{b}(t) & =\frac{m_{b}(t) R_{g} T}{V_{b_{0}}+A_{p} x(t)}
\end{aligned}
$$

where we assume an isothermal process in which the (ideal) gas temperature is constant at $T, R_{g}$ is the gas constant for the pneumatic gas, and $V_{t_{0}}$ and $V_{b_{0}}$ are the minimum gas volumes for the gas chambers above and below the piston, respectively.

The gas flows are given by:

$$
\begin{aligned}
& f_{t}(t)=f_{g}\left(p_{t}(t), u_{t}(t)\right) \\
& f_{b}(t)=f_{g}\left(p_{b}(t), u_{b}(t)\right)
\end{aligned}
$$

where $f_{g}$ defines gas flow through an orifice for choked and non-choked flow conditions [19]: $f_{g}\left(p_{1}, p_{2}\right)=$

$$
\left\{\begin{array}{c}
C_{s} A_{s} p_{1} \sqrt{\frac{\gamma}{Z R_{g} T}\left(\frac{2}{\gamma+1}\right)^{\frac{\gamma+1}{\gamma-1}}}, \\
p_{1} \geq p_{2} \wedge p_{1} / p_{2} \geq\left(\frac{\gamma+1}{2}\right)^{\gamma /(\gamma-1)} \\
C_{s} A_{s} p_{1} \sqrt{\frac{\gamma}{Z R_{g} T}\left(\frac{2}{\gamma-1}\right)\left(\left(\frac{p_{2}}{p_{1}}\right)^{\frac{2}{\gamma}}-\left(\frac{p_{2}}{p_{1}}\right)^{\frac{\gamma+1}{\gamma}}\right)}, \\
C_{s} A_{s} p_{2} \sqrt{\frac{p_{1} \geq p_{2} \wedge p_{1} / p_{2}<\left(\frac{\gamma+1}{2}\right)^{\gamma /(\gamma-1)}\left(\frac{2}{\gamma+1}\right)^{\frac{\gamma+1}{\gamma-1}}}{Z R_{g} T}} \\
C_{s} A_{s} p_{2} \sqrt{\frac{p_{1}}{Z R_{g} T}\left(\frac{2}{\gamma-1}\right)\left(\left(\frac{p_{1}}{p_{2}}\right)^{\frac{2}{\gamma}}-\left(\frac{p_{1}}{p_{2}}\right)^{\frac{\gamma+1}{\gamma}}\right)} \\
p_{1}<p_{2} \wedge p_{2} / p_{1}<\left(\frac{\gamma+1}{2}\right)^{\gamma /(\gamma-1)}
\end{array}\right.
$$

where $\gamma$ is the ratio of specific heats, $Z$ is the gas compressibility factor, $C_{s}$ is the flow coefficient, and $A_{s}$ is the orifice area. Choked flow occurs when the pressure ratio exceeds $\left(\frac{\gamma+1}{2}\right)^{\gamma /(\gamma-1)}$.

We select our complete measurement vector as

$$
\mathbf{y}(t)=\left[\begin{array}{c}
x(t) \\
p_{t}(t) \\
p_{b}(t) \\
f_{v}(t) \\
\operatorname{open}(t) \\
\operatorname{closed}(t)
\end{array}\right],
$$

where $f_{v}$ is the fluid flow through the valve:

$$
f_{v}(t)=\frac{x(t)}{L_{s}} C_{v} A_{v} \sqrt{\frac{2}{\rho}\left|p_{f l}-p_{f r}\right|} \operatorname{sign}\left(p_{f l}-p_{f r}\right),
$$

$C_{v}$ is the (dimensionless) flow coefficient of the valve, $\rho$ is the liquid density, and we assume a linear flow characteristic for the valve. The open $(t)$ and closed $(t)$ measurements are discrete sensors which output 1 if the valve is in the fully opened or fully closed state:

$$
\begin{aligned}
\operatorname{open}(t) & = \begin{cases}1, & \text { if } x(t) \geq L_{s} \\
0, & \text { otherwise }\end{cases} \\
\operatorname{closed}(t) & = \begin{cases}1, & \text { if } x(t) \leq 0 \\
0, & \text { otherwise }\end{cases}
\end{aligned}
$$

It should be noted how the discrete position sensors (open and closed) are treated within the particle filter framework. In a particle filter, a certain amount of sensor noise must 
be assumed for sensors, but, in practice, the discrete position sensors have no noise. If an artificial amount of noise is not assumed within the particle filter for these sensors, then sever degeneracy will result, because very few, if any, particles will be able to predict the transitions of these sensor values. The degeneracy is severe enough that it cannot be remedied by resampling methods. Assuming some amount of sensor noise addresses this issue.

Fig. 3 shows a nominal valve cycle. The valve is commanded to open at $0 \mathrm{~s}$. The top pneumatic port opens to atmosphere and the bottom opens to the supply pressure (approximately $5.3 \mathrm{MPa}$, or $750 \mathrm{psig}$ ). When the force on the underside of the piston is large enough to overcome the return spring, friction, and the gas force on the top of the piston, the valve begins to move upward as the pneumatic gas continues to flow into and out of the valve actuator. At about $8 \mathrm{~s}$ the valve is completely open. The valve is commanded to close at $15 \mathrm{~s}$. The bottom pneumatic port opens to atmosphere and the bottom opens to the supply pressure. When the force balance becomes negative, the valve starts to move downward, and completely closes at around $20 \mathrm{~s}$. The valve closes faster than it opens due to the return spring.

\section{B. Damage Modeling}

Our general approach to damage modeling is as follows. First, we identify parameters in the model that characterize the extent of specific forms of damage, and these augment the state vector $\mathbf{x}$. We then incorporate models of how those parameters change over time with system operation. It is the parameters of these equations that are unknown and must be estimated, and also augment $\mathbf{x}$ for that purpose. In the valve model, we consider damage or wear characterized by the increase in friction coefficient, the decrease in spring constant, the appearance and growth of an internal valve leak between the volumes on either side of the piston, and the appearance and growth of external leaks at the pneumatic ports.

One damage mechanism present in valves is sliding wear. The equation for sliding wear takes on the following form [20]:

$$
\dot{V}(t)=w|F(t) v(t)|,
$$

where $V(t)$ is the wear volume, $w$ is the wear coefficient (which depends on material properties such as hardness), $F(t)$ is the sliding force, and $v(t)$ is the sliding velocity. Friction will increase linearly with sliding wear, because the contact area between the sliding bodies becomes greater as surface asperities wear down [20]. Lubrication between the sliding bodies can also degrade over time. We therefore model the change in friction coefficient in a form similar to sliding wear:

$$
\dot{r}(t)=w_{r}\left|F_{f}(t) v(t)\right|
$$

where $w_{r}$ is the wear coefficient, and $F_{f}(t)$ is the friction force defined in the previous subsection. Fig. 4 shows the effect of an increase in friction on the valve cycle. From the simulation, we can determine the value of the friction parameter, $r^{*}$, at which the valve has reached EOL. At this value, the friction force becomes large enough that the valve cannot open within
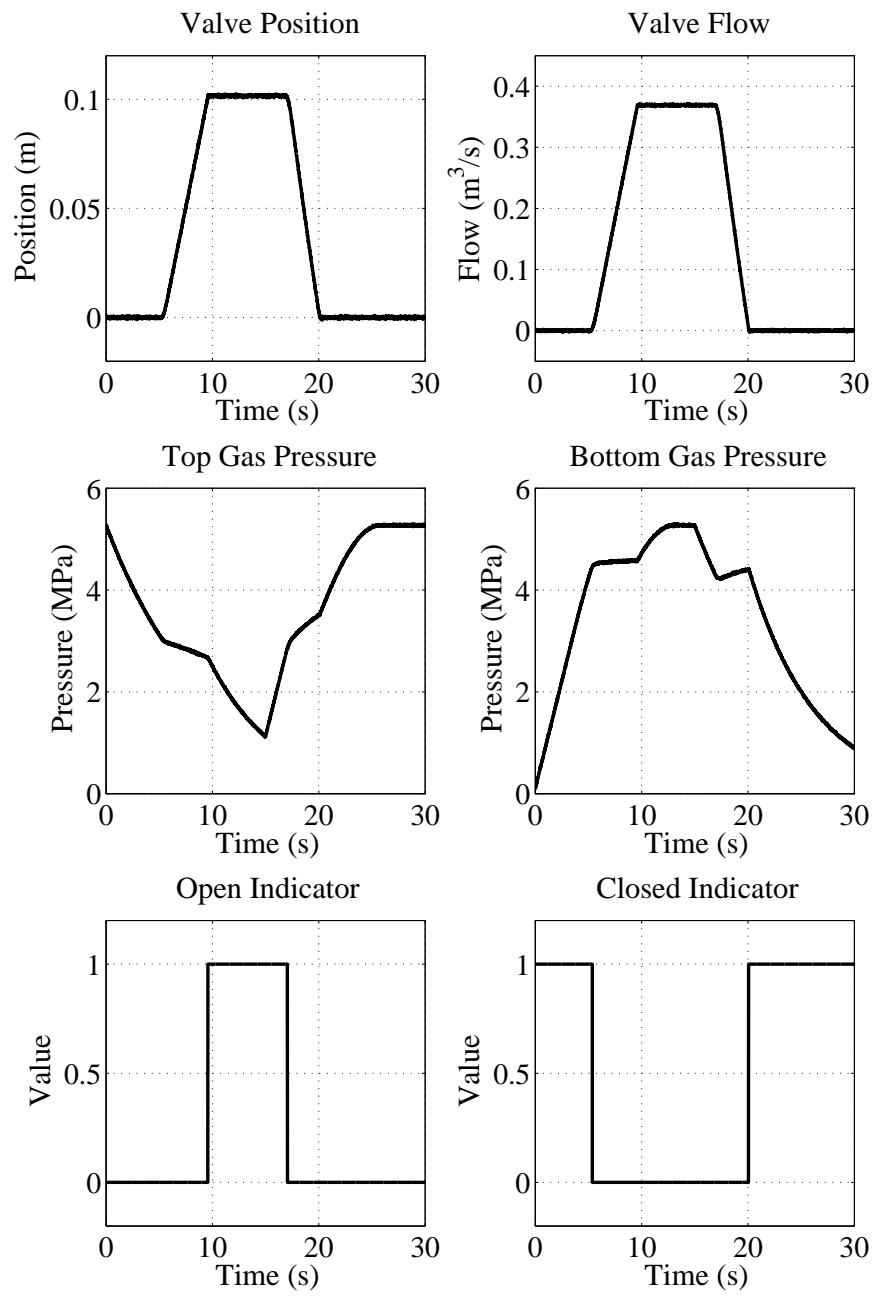

Fig. 3. Nominal valve operation.

the $15 \mathrm{~s}$ limit, as shown in Fig. 4. So, $C_{E O L}(\mathbf{x}(t), \boldsymbol{\theta}(t))=1$ if $r(t) \geq r^{*}$.

We assume the same equation form for spring damage:

$$
\dot{k}(t)=-w_{k}\left|F_{s}(t) v(t)\right|,
$$

where $w_{k}$ is the spring wear coefficient and $F_{s}(t)$ is the spring force. The more the spring is used, the weaker it becomes. We define $k^{*}$ as the largest value of $k$ at which the valve will not fully close upon loss of supply pressure. Fig. 4 shows the effect of a decrease in the spring parameter on the valve cycle. In normal operation, without the spring tending the valve to close, the valve will open faster and close slower. However, the spring must be strong enough to close the valve against system pressure when the actuating pressure is lost. So, $C_{E O L}(\mathbf{x}(t), \boldsymbol{\theta}(t))=1$ also if $k(t) \leq k^{*}$.

An internal leak in the valve can appear at the seal surrounding the piston as a result of sliding wear. The pneumatic gas is then able to flow between the volumes above and below the piston, decreasing the response time of the valve. We parameterize this leak by its equivalent orifice area, $A_{i}(t)$, 

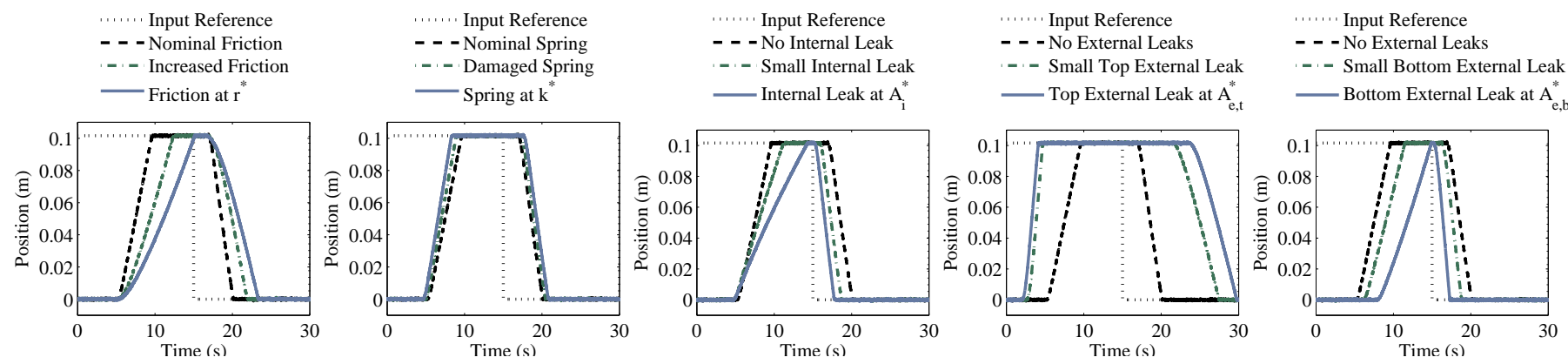

Fig. 4. Valve operation with increasing wear.

described by:

$$
\dot{A}_{i}(t)=w_{i}\left|F_{f}(t) v(t)\right|,
$$

where $w_{i}$ is the wear coefficient. The mass flow at the leak, $f_{i}(t)$, is computed using the gas flow equation:

$$
f_{i}(t)=f_{g}\left(p_{t}(t), p_{b}(t)\right) .
$$

As sliding wear occurs, the leak size keeps increasing. The presence of an internal leak makes it more difficult to actuate the valve, because it causes gas to flow into the lower pressure volume that is being evacuated and out of the higher pressure volume that is being filled. We define $A_{i}^{*}$ as the minimum internal leak area at which the valve cannot open within the 15 s limit. So, $C_{E O L}(\mathbf{x}(t), \boldsymbol{\theta}(t))=1$ also if $A_{i}(t) \geq A_{i}^{*}$. Fig. 4 shows the effect of an internal leak on the valve cycle.

External leaks can also form, most likely at the actuator connections to the pneumatic gas supply, due to corrosion and other environmental factors. Without knowledge of how the leak size progresses, we assume the growth of the area of the leak holes, $A_{e}(t)$, is linear:

$$
\dot{A}_{e}(t)=w_{e}
$$

where $w_{e}$ is the wear coefficient. We use additional $t$ and $b$ subscripts to denote leaks at the top and bottom pneumatic ports, respectively. The effect of the formation of a leak at the top pneumatic port is that it becomes easier to open the valve but more difficult to close it. Conversely, the effect of a leak at the bottom pneumatic port is that it becomes more difficult to open but easier to close the valve. Through simulation we can determine the minimum size leak holes at which the valve cannot open or close within the $15 \mathrm{~s} \mathrm{limit}, A_{e, t}^{*}$ and $A_{e, b}^{*}$. (An alternative is to use a maximum allowable leakage rate to define EOL.) So, $C_{E O L}(\mathbf{x}(t), \boldsymbol{\theta}(t))=1$ also if $A_{e, t}(t) \geq A_{e, t}^{*}$ or $A_{e, b}(t) \geq A_{e, b}^{*}$. Fig. 4 shows the effect of external leak on the valve cycle.

\section{Experimental Results}

We performed a number of simulation experiments to validate our prognostics methodology and evaluate how it performs under different sensor sets using the prognostics performance metrics described in [13], [14]. In each experiment, we considered additive zero-mean process and measurement noise, used $N=500$ particles, and used a sample time of

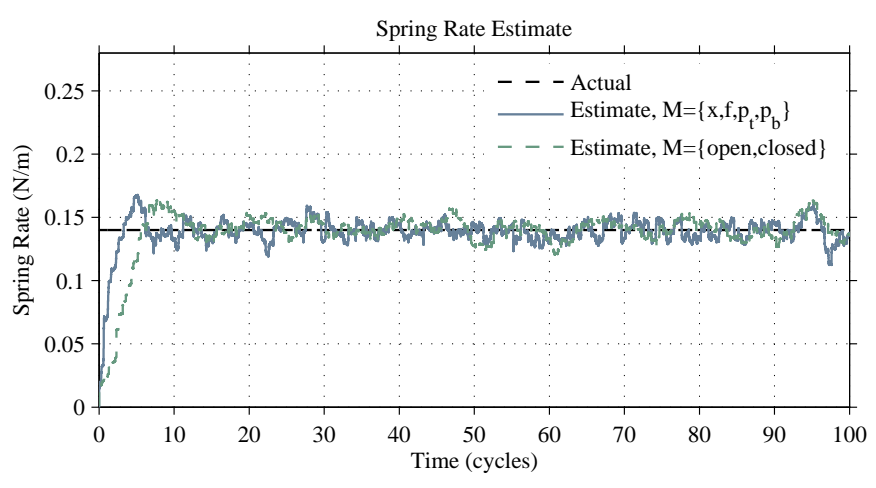

Fig. 5. Estimation results for $w_{k}$ with $M=\left\{x, f, p_{t}, p_{b}\right\}$ and $M=$ $\{$ open, closed $\}$.

$0.01 \mathrm{~s}$. We assumed that only a single damage mechanism was active, and, in each experiment, started from the point where damage has been identified and the only unknown is the wear coefficient (initially assumed to be 0 for parameter estimation). The wear coefficients were chosen so that EOL would be reached between 100 and 150 cycles.

The particle filters must be tuned to use the appropriate amount of noise. Assuming the variance of the process was known, the particle filters were set to sample with the same amount of process noise. The random walk variances were selected assuming the orders of magnitude of the wear coefficients were known. The particle filters were also set to assume 10 times the amount of actual measurement noise (assumed to be known). For the discrete position sensors, a value of 0.1 for the variance of these sensors was used, which resulted in the best overall estimation performance for these scenarios. With these settings fixed, we then varied only the measurement set, denoted as $M$.

First, we provide an example scenario that demonstrates our model-based methodology and explains the metrics used to evaluate performance. We consider the case of spring damage, where $w_{k}=0.14, E O L^{*}=106.6$ cycles (where EOL $L^{*}$ and $R U L^{*}$ denote the true EOL and RUL), and examine two cases in detail: one with $M=\left\{x, f, p_{t}, p_{b}\right\}$, and one with $M=\{$ open, closed $\}$. Fig. 5 shows the estimation of the hidden parameter, $w_{k}$ for these two cases. As observed, the estimates converge then track the true wear parameter value. 
The two cases appear to have comparable estimation accuracy. Clearly though, the $M=\{$ open, closed $\}$ case is slower to converge, achieving convergence within 15 cycles, whereas the $M=\left\{x, f, p_{t}, p_{b}\right\}$ converges within 10 . We evaluate the performance of the wear parameter estimation using three metrics, for quantifying accuracy, spread, and convergence.

Accuracy is calculated using the percentage root mean square error (PRMSE), which expresses relative estimation accuracy as a percentage:

$$
\operatorname{PRMSE}=100 \sqrt{\operatorname{Mean}_{k}\left[\left(\frac{\hat{w}_{k}-w_{k}^{*}}{w_{k}^{*}}\right)^{2}\right]},
$$

where $\hat{w}_{k}$ denotes the estimated wear parameter value at time $k, w_{k}^{*}$ denotes the true wear parameter value at $k$, and Mean $_{k}$ denotes the mean over all values of $k$. In computing PRMSE, we ignore the first 10 cycles (300 s), where most of the error is associated with convergence. In this case, the PRMSE is $5.11 \%$ for $M=\left\{x, f, p_{t}, p_{b}\right\}$, and $5.23 \%$ for $M=\{$ open, closed $\}$.

We calculate the spread using the relative median absolute deviation (RMAD), which expresses the spread relative to the median as a percentage:

$$
\operatorname{RMAD}(X)=100 \frac{\operatorname{Median}_{i}\left(\left|X_{i}-\operatorname{Median}_{j}\left(X_{j}\right)\right|\right)}{\operatorname{Median}_{j}\left(X_{j}\right)},
$$

where $X$ is a data set and $X_{i}$ is an element of that set. For estimation spread, for time $k$ we use the distribution of wear parameter values given by the particle set at $k$ as the data set. Note that since we use the SIR filter, all particles are equally weighted and the median can be directly applied. We denote the average RMAD over multiple $k$ using $\overline{R M A D}_{w}$ :

$$
\overline{\operatorname{RMAD}}_{w}=\operatorname{Mean}_{k}\left(\operatorname{RMAD}_{w, k}\right),
$$

where $\operatorname{RMAD}_{w, k}$ denotes the RMAD of the wear parameter at time $k$. In this example, $\overline{\operatorname{RMAD}}_{w}=11.00 \%$ for $M=\left\{x, f, p_{t}, p_{b}\right\}$ and $\overline{\mathrm{RMAD}}_{w}=16.23 \%$ for $M=$ $\{$ open, closed $\}$. Although estimation accuracy is comparable, the spread is significantly better when using the full set of continuous sensors.

The final estimation metric is convergence of the wear parameter estimate, denoted as $C_{w}$. We use the definition of the convergence metric described in [13], where the convergence of a curve is expressed as the distance from the origin to the centroid under the curve (a shorter distance is better). We use the absolute error of the hidden parameter estimate as the curve. In this case, $C_{w}=27.99$ for $M=\left\{x, f, p_{t}, p_{b}\right\}$, and $C_{w}=44.04$ for $M=\{$ open, closed $\}$. Since the wear parameter is many orders of magnitude smaller than the time scale, the convergence score is approximately in units of seconds. We take convergence only over the first 100 s so that errors after convergence do not contribute. Otherwise, a curve with fast convergence but poor tracking could score higher than a curve with slow convergence but excellent tracking. The poor tracking in the former case is captured using the PRMSE metric.
High estimation performance should translate to high prediction performance if future inputs are known. In the case of the pneumatic valve, the inputs that describe a complete valve cycle are known, so there is no uncertainty in input prediction for our purposes. Therefore, a low $C_{w}$ score means that accurate predictions are available sooner, i.e., a prognostics horizon is arrived at earlier. A low PRMSE score means that on average, estimates of the wear rate are closer to the true value, so point predictions of EOL and RUL will on average be closer to their true values. Accordingly, a low $\overline{\mathrm{RMAD}}_{w}$ score translates to more precise predictions.

We summarize prediction performance in the $\alpha-\lambda$ metric [13], [14]. Here, $\alpha \in[0,1]$ defines bounds as a function of the true RUL, i.e., $(1 \pm \alpha) R U L^{*}$, and $\lambda \in[0,1]$ denotes the fraction of the time from the first prediction to the true EOL. We use the extended version of the metric, which incorporates a third parameter, $\beta \in[0,1]$, which defines a bound on the fraction of the probability mass of a prediction that falls within the $\alpha$-bounds [14]. The $\alpha-\lambda$ metric requires that for given prediction times, a desired portion of the predicted RUL distribution fits within a cone of accuracy defined by $\alpha$. The $\alpha-\lambda$ plot for $M=\left\{x, f, p_{t}, p_{b}\right\}$ is shown in Fig. 6, where $\alpha=0.1$ and $\beta=0.4$ for all $\lambda$ (all $k_{P}$ ). The distribution at each prediction point is represented using a box plot. The percentage of the probability mass that falls within the $\alpha$ bounds is shown above each box plot, along with the result of the metric at that point (true or false). As shown in the figure, the desired performance criteria are met at each prediction point except for the 60-cycle prediction point. Note, however, that at this point, the median prediction is within the $\alpha$-bounds, and the mean is only just outside. With $\alpha=0.11$, the metric is satisfied at all prediction points. Fig. 7 shows the $\alpha-\lambda$ plot for $M=\{$ open, closed $\}$. Although the mean and median are typically within the $\alpha$-bounds, on average only about $32 \%$ of the probability mass is contained within the bounds. With $\alpha=0.18$, the metric is satisfied at all prediction points.

For a particular prediction point $k_{P}$, we compute measures of accuracy and prediction. For accuracy, we use the relative accuracy (RA) metric:

$$
\mathrm{RA}_{k_{P}}=1-\frac{\left|R U L_{k_{P}}^{*}-\operatorname{Median}_{i}\left(R U L_{k_{P}}^{i}\right)\right|}{R U L_{k_{P}}^{*}},
$$

Here, we have chosen the median as the measure of central tendency of the distribution. The mean could also be used, but we have found the median to be more accurate in our experiments. We calculate prediction spread using RMAD, which we denote as $\operatorname{RMAD}_{R U L}$. As a second measure of spread, we compute the fraction of the probability mass that falls within specified $\alpha$-bounds, denoted as $\pi_{\alpha}$. To summarize these metrics over a particular experiment, we average them over all prediction points. We use $\overline{\mathrm{RA}}$ to denote the averaged RA, $\overline{\mathrm{RMAD}}_{R U L}$ to denote averaged RMAD, and $\bar{\pi}_{\alpha}$ to denote the average fraction of the probability within the $\alpha$-bounds. In the current example, for $M=\left\{x, f, p_{t}, p_{b}\right\}, \overline{R A}=0.953, \bar{\pi}_{\alpha}=$ $0.487, \overline{\operatorname{RMAD}}_{R U L}=10.23 \%$, and for $M=\{$ open, closed $\}$, $\overline{\mathrm{RA}}=0.934, \bar{\pi}_{\alpha}=0.326, \overline{\mathrm{RMAD}}_{R U L}=16.16 \%$. We also 


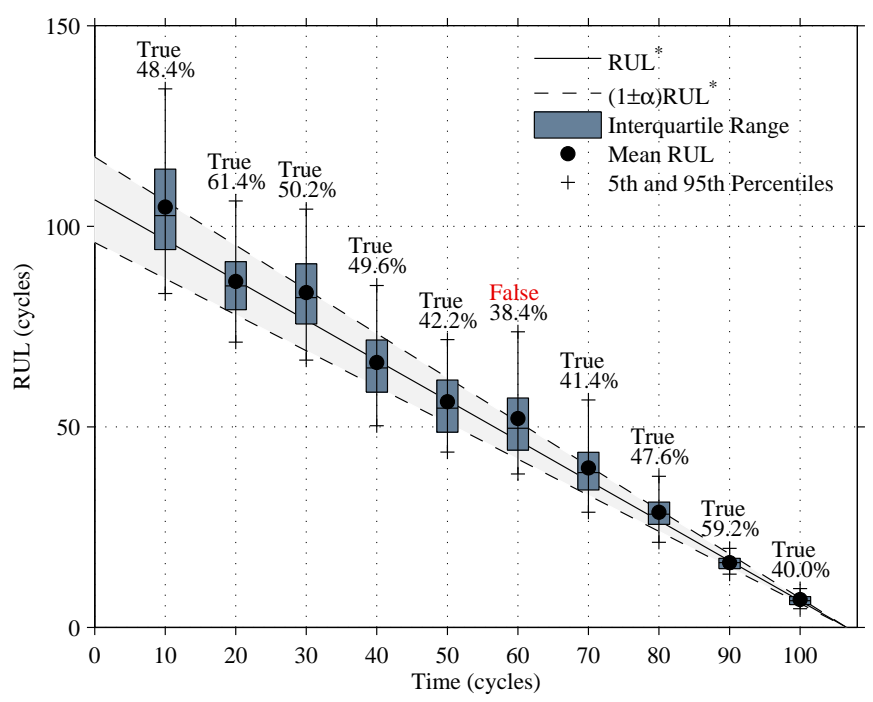

Fig. 6. $\alpha-\lambda$ metric for spring damage prediction, where $\alpha=0.1, \beta=0.4$, and $M=\left\{x, f, p_{t}, p_{b}\right\}$.

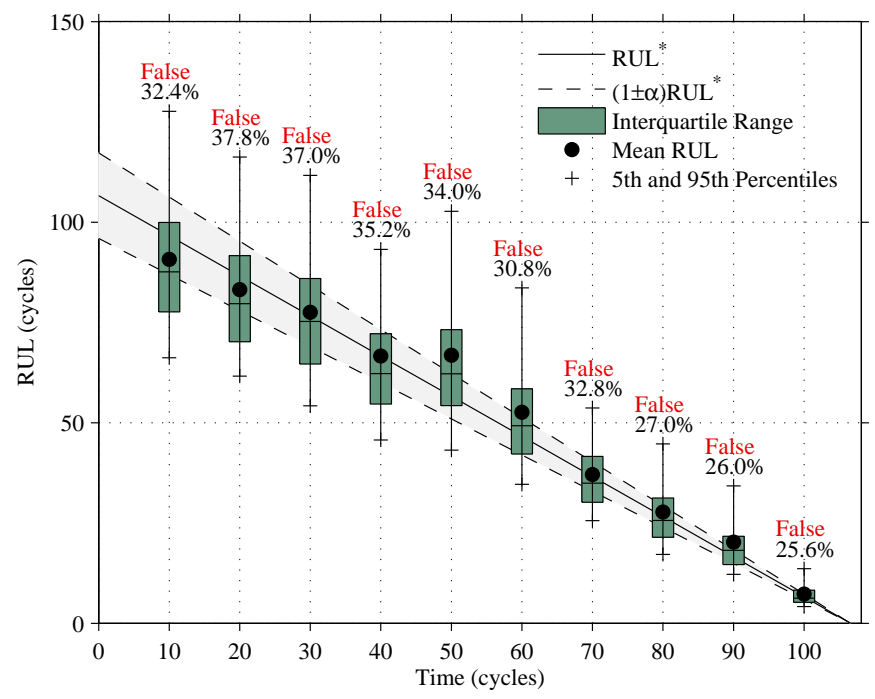

Fig. 7. $\alpha-\lambda$ metric for spring damage prediction, where $\alpha=0.1, \beta=0.4$, and $M=\{$ open, closed $\}$

consider worst-case performance over all prediction points. We use the ${ }^{-}$superscript to denote a minimum and the ${ }^{+}$ superscript to denote a maximum. For $M=\left\{x, f, p_{t}, p_{b}\right\}$, $R A^{-}=0.927, \pi_{\alpha}^{-}=0.384$, and $\operatorname{RMAD}_{R U L}^{+}=14.81 \%$. For $M=\{$ open, closed $\}, \mathrm{RA}^{-}=0.901, \pi_{\alpha}^{-}=0.326$, and $\mathrm{RMAD}_{R U L}^{+}=21.92 \%$. In these cases, a clear connection is observable between performance in estimation and performance in prediction (e.g., for $M=\left\{x, f, p_{t}, p_{b}\right\}$, PRMSE $=$ $5.11 \%$ where $\overline{\mathrm{RA}}=0.953$, and $\overline{\mathrm{RMAD}}_{w}=11.00 \%$ where $\overline{\mathrm{RMAD}}_{R U L}=10.23 \%$. Both sensor sets have fairly good accuracy, but using $M=\left\{x, f, p_{t}, p_{b}\right\}$ achieves significantly better precision.

We now present a comprehensive set of simulation results for each sensor set for all considered faults. The results are shown in Table I. We report PRMSE of the wear parameter (in \%), $\overline{\operatorname{RMAD}}_{w}$ (in \%), $C_{w}$ (approximately in seconds), $\overline{\mathrm{RA}}, \mathrm{RA}^{-}, \bar{\pi}_{\alpha}$ for $\alpha=0.1, \pi_{\alpha}^{-}, \overline{\mathrm{RMAD}}_{R U L}$ (in $\%$ ), and $\mathrm{RMAD}_{R U L}^{+}$(in \%). The results presented are averaged over 10 experiments per scenario.

Overall, the expected results are obtained. The PRMSE is under $10 \%$ for all cases, and is around $5 \%$ for most. Correspondingly, RA is, in most cases, above $95 \%$. The $\bar{\pi}_{\alpha}$ score is typically desired to be at least $50 \%$, but here it is lower. For some faults, the prediction variance is large because the random walk variances were not optimally tuned. In general, this cannot be done beforehand since wear parameters are unknown. In an online setting however, correction loops can be used to reduce this variance over time [9].

In general, it is observed that not much difference exists in relative accuracy over the different sensor sets. In fact, in some cases, estimation of the wear parameter is better using only the discrete position sensors rather than the full set of continuous measurements. This means that accurate point predictions can still be achieved even under limited sensing scenarios. However, the prediction spread is significantly larger with limited sensor sets. This is observed best using $\bar{\pi}_{\alpha}$. For example, for the bottom external leak, there is a $27 \%$ decrease from the full set of continuous measurements to the sensor set containing only the discrete position sensors. Convergence of the wear parameter estimate is also affected, although there is no significant difference for the top external leak, which we attribute to the fact that a top external leak of the selected wear rate is easily visible in the pressure measurements, so convergence is quickly forced for all choices of $M$.

The results also demonstrate the usefulness of different sensors. The flow measurement effectively provides a redundant position measurement, so dropping either $x$ or $f$ from the sensor set has little effect on performance. Some sensors also give more useful information on specific faults, as observed in the cases were $M$ is a singleton. For changes in the friction coefficient and spring rate, $M=\{x\}$ outperforms $\left\{p_{t}\right\},\left\{p_{b}\right\}$, and also $\left\{p_{t}, p_{b}\right\}$. This is because the position measurement gives more direct information on changes in friction. Similarly, for the cases with leaks, the pressure measurements are more useful. For the internal leak and bottom external leak, $p_{b}$ is most useful, and for the top external leak, $p_{t}$ is most useful.

As observed, even by just using the discrete position sensors, accurate point predictions can still be made. In addition to the uncertainty management capabilities of particle filters, this robust performance is largely due to having a good model of the component and its wear mechanisms, which is why we favor model-based approaches. To investigate how the performance changes when both measurements and the model are less reliable, we ran a set of experiments with increased process and measurement noise. The results are presented in Table II for the bottom leak fault. We evaluate performance using the key metrics PRMSE, $\overline{\mathrm{RA}}, \bar{\pi}_{\alpha}$ for $\alpha=0.1$, and $\overline{\mathrm{RMAD}}_{R U L}$. Results were averaged over 15 runs for each choice of $M$. The baseline noise level is denoted by $\xi$, which 
TABLE I

AVERAGE PERFORMANCE FOR EACH FAULT UNDER DIFFERENT SENSOR SETS

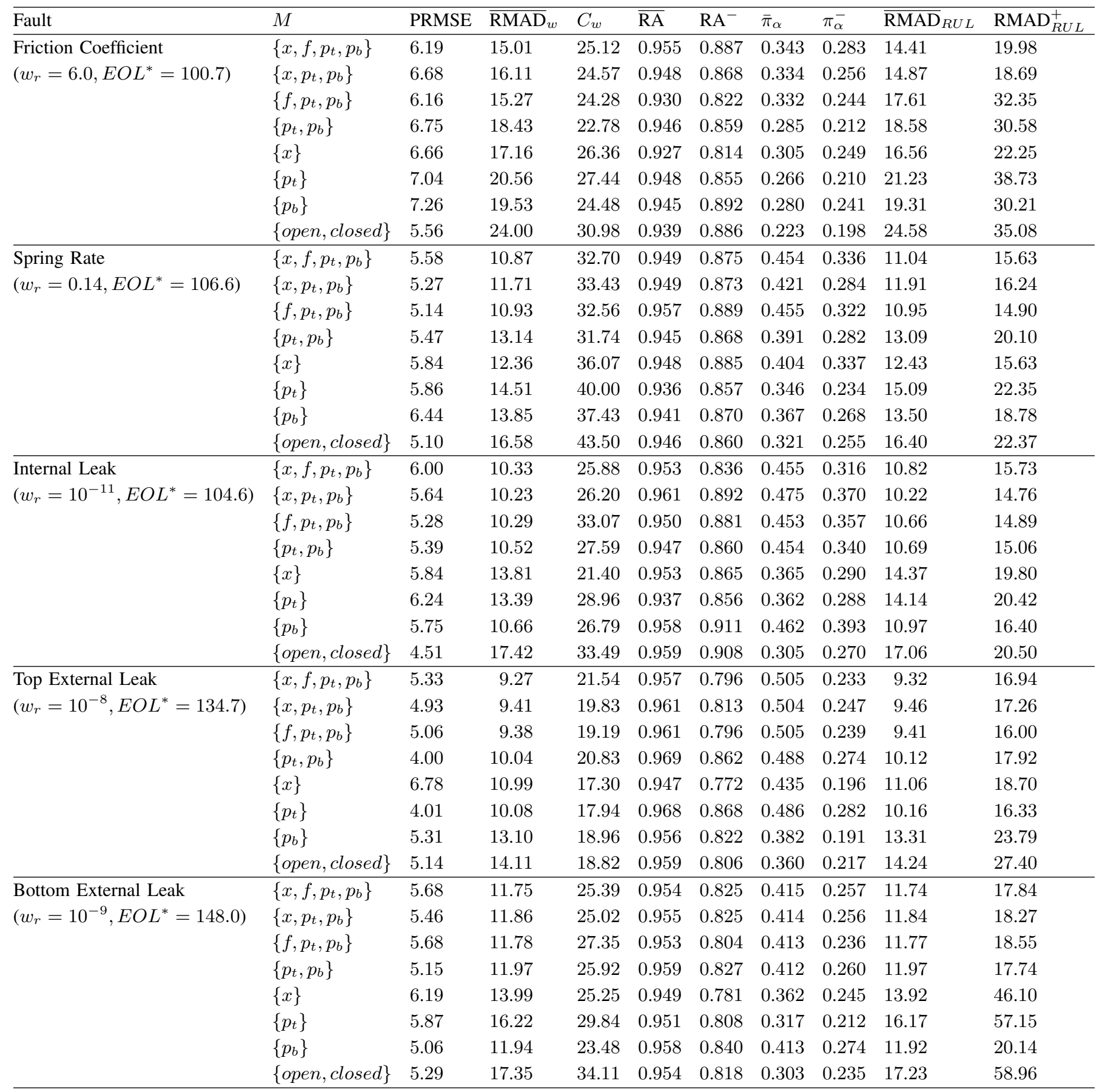


TABLE II

AVERAGE PERFORMANCE FOR THE BOTTOM LEAK FAULT UNDER DIFFERENT NOISE LEVELS

\begin{tabular}{|c|c|c|c|c|c|c|c|c|c|c|c|c|}
\hline \multirow[t]{2}{*}{$\bar{M}$} & \multicolumn{3}{|c|}{ PRMSE } & \multicolumn{3}{|c|}{$\overline{\overline{\mathrm{RA}}}$} & \multicolumn{3}{|c|}{$\bar{\pi}_{\alpha}$} & \multicolumn{3}{|c|}{$\overline{\operatorname{RMAD}}_{R U L}$} \\
\hline & $\xi$ & $10 \xi$ & $100 \xi$ & $\xi$ & $10 \xi$ & $100 \xi$ & $\xi$ & $10 \xi$ & $100 \xi$ & $\xi$ & $10 \xi$ & $100 \xi$ \\
\hline$\left\{x, f, p_{t}, p_{b}\right\}$ & 5.68 & 8.60 & 12.87 & 0.954 & 0.931 & 0.899 & 0.415 & 0.355 & 0.275 & 11.74 & 13.86 & 18.14 \\
\hline$\left\{x, p_{t}, p_{b}\right\}$ & 5.46 & 7.77 & 11.27 & 0.955 & 0.935 & 0.907 & 0.413 & 0.358 & 0.274 & 11.84 & 13.75 & 18.36 \\
\hline$\left\{f, p_{t}, p_{b}\right\}$ & 5.68 & 7.94 & 11.98 & 0.953 & 0.935 & 0.902 & 0.413 & 0.357 & 0.279 & 11.77 & 13.90 & 18.02 \\
\hline$\left\{p_{t}, p_{b}\right\}$ & 5.15 & 7.20 & 10.97 & 0.959 & 0.939 & 0.909 & 0.411 & 0.359 & 0.271 & 11.97 & 13.96 & 18.60 \\
\hline$\{x\}$ & 6.19 & 8.39 & 12.49 & 0.949 & 0.932 & 0.896 & 0.362 & 0.302 & 0.233 & 13.92 & 17.45 & 22.66 \\
\hline$\left\{p_{t}\right\}$ & 5.87 & 9.03 & 14.63 & 0.951 & 0.920 & 0.874 & 0.316 & 0.249 & 0.190 & 16.17 & 21.20 & 27.68 \\
\hline$\left\{p_{b}\right\}$ & 5.06 & 7.79 & 10.29 & 0.958 & 0.935 & 0.915 & 0.412 & 0.349 & 0.278 & 11.92 & 14.26 & 18.72 \\
\hline$\{$ open, closed $\}$ & 5.29 & 7.03 & 11.69 & 0.954 & 0.941 & 0.904 & 0.303 & 0.284 & 0.241 & 17.23 & 19.12 & 21.76 \\
\hline
\end{tabular}

corresponds to about $0.1 \%$ process noise and $1 \%$ measurement noise. We increased the noise variances by factors of 10 (denoted by $10 \xi$ ) and 100 (denoted by $100 \xi$ ). The $100 \xi$ noise level corresponds to $1 \%$ process noise and $10 \%$ measurement noise.

As shown in the table, performance degrades as noise is increased. However, even with $100 \xi$, the average RA has only decreased to about $90 \%$ for all choices of $M$. Significant reductions in prediction spread are also observed. The difference in performance between the full sensor set and using only the discrete position sensors, though, is reduced in the higher noise scenarios. Performance is the best when the bottom pressure sensor is used, as this provides the most direct information for the bottom leak fault.

\section{CONCLUSIONS}

In this paper, we developed a general model-based prognostics methodology using particle filters, formulated as a joint state-parameter estimation problem. State-parameter estimates are propagated forward in time to obtain EOL and RUL predictions, based on models that capture the progression of damage over time, characterized by a set of unknown parameters. We evaluated the performance under limited sensor sets using prognostics performance metrics [13]. It was shown that, with an accurate model, accurate predictions can still be made even when only discrete position sensors are utilized. The disadvantage is that prediction spread may increase significantly. We also investigated how performance degrades when model uncertainty (i.e., process noise) and sensor noise are increased. Even with the maximum amount of noise considered, an average of $90 \%$ accuracy was still achievable, although under much wider prediction spread.

In future work, we will investigate how methods to decrease prediction spread such as correction loops [9] and fixed-lag filters [5] can improve performance under limited sensor sets. In addition, we assumed only single damage mechanisms were active in our experiments. While the approach presented can still be applied to estimation of multiple wear parameters, a decrease in performance is expected. Investigating that case under limited sensor sets is also of interest.

\section{ACKNOWLEDGMENTS}

The funding for this work was provided by the NASA Fault Detection, Isolation, and Recovery (FDIR) project under the Exploration Technology and Development Program (ETDP) of the Exploration Systems Mission Directorate (ESMD).

\section{REFERENCES}

[1] M. Schwabacher, "A survey of data-driven prognostics," in Proceedings of the AIAA Infotech@ Aerospace Conference, 2005.

[2] M. Roemer, C. Byington, G. Kacprzynski, and G. Vachtsevanos, "An overview of selected prognostic technologies with reference to an integrated PHM architecture," in Proceedings of the First International Forum on Integrated System Health Engineering and Management in Aerospace, 2005.

[3] C. S. Byington, M. Watson, D. Edwards, and P. Stoelting, "A modelbased approach to prognostics and health management for flight control actuators," in Proceedings of the 2004 IEEE Aerospace Conference, vol. 6, March 2004, pp. 3551-3562.

[4] B. Saha and K. Goebel, "Modeling Li-ion battery capacity depletion in a particle filtering framework," in Proceedings of the Annual Conference of the Prognostics and Health Management Society 2009, Sept. 2009.

[5] M. Daigle and K. Goebel, "Model-based prognostics with fixed-lag particle filters," in Proceedings of the Annual Conference of the Prognostics and Health Management Society 2009, Sept. 2009.

[6] M. Abbas, A. A. Ferri, M. E. Orchard, and G. J. Vachtsevanos, "An intelligent diagnostic/prognostic framework for automotive electrical systems," in 2007 IEEE Intelligent Vehicles Symposium, 2007, pp. 352357.

[7] E. Bechhoefer, "A method for generalized prognostics of a component using Paris law," in Proceedings of 64th American Helicopter Society Annual Forum, Apr. 2008.

[8] F. Cadini, E. Zio, and D. Avram, "Monte Carlo-based filtering for fatigue crack growth estimation," Probabilistic Engineering Mechanics, vol. 24, no. 3, pp. 367-373, 2009.

[9] M. Orchard, G. Kacprzynski, K. Goebel, B. Saha, and G. Vachtsevanos, "Advances in uncertainty representation and management for particle filtering applied to prognostics," in Proceedings of International Conference on Prognostics and Health Management, Oct. 2008.

[10] P. Li, R. Goodall, P. Weston, C. Seng Ling, C. Goodman, and C. Roberts, "Estimation of railway vehicle suspension parameters for condition monitoring," Control Engineering Practice, vol. 15, no. 1, pp. 43-55, 2007.

[11] B. Saha, K. Goebel, S. Poll, and J. Christophersen, "Prognostics methods for battery health monitoring using a bayesian framework," IEEE Transactions on Instrumentation and Measurement, vol. 58, no. 2, pp. 291-296, Feb. 2009.

[12] M. E. Orchard, L. Tang, K. Goebel, and G. Vachtsevanos, "A novel RSPF approach to prediction of high-risk, low-probability failure events," in Proceedings of the Annual Conference of the Prognostics and Health Management Society 2009, Sept. 2009. 
[13] A. Saxena, J. Celaya, E. Balaban, K. Goebel, B. Saha, S. Saha, and M. Schwabacher, "Metrics for evaluating performance of prognostic techniques," in International Conference on Prognostics and Health Management 2008, Oct 2008.

[14] A. Saxena, J. Celaya, B. Saha, S. Saha, and K. Goebel, "On applying the prognostic performance metrics," in Proceedings of the Annual Conference of the Prognostics and Health Management Society 2009, Sept. 2009.

[15] M. S. Arulampalam, S. Maskell, N. Gordon, and T. Clapp, "A tutorial on particle filters for online nonlinear/non-Gaussian Bayesian tracking," IEEE Transactions on Signal Processing, vol. 50, no. 2, pp. 174-188, 2002.

[16] O. Cappe, S. J. Godsill, and E. Moulines, “An overview of existing methods and recent advances in sequential Monte Carlo," Proceedings of the IEEE, vol. 95, no. 5, p. 899, 2007.

[17] G. Kitagawa, "Monte Carlo filter and smoother for non-Gaussian nonlinear state space models," Journal of Computational and Graphical Statistics, vol. 5, no. 1, pp. 1-25, 1996.

[18] A. Doucet, S. Godsill, and C. Andrieu, "On sequential Monte Carlo sampling methods for Bayesian filtering," Statistics and Computing, vol. 10, pp. 197-208, 2000.

[19] R. Perry and D. Green, Perry's chemical engineers' handbook. McGraw-Hill Professional, 2007.

[20] I. M. Hutchings, Tribology: friction and wear of engineering materials. CRC Press, 1992. 